\title{
SOUNDFIELD REPRODUCTION USING THEORETICAL CONTINUOUS LOUDSPEAKER
}

\author{
Yan Jennifer WU \& Thushara D. Abhayapala \\ Dept of Information Engineering, Research School of Information Sciences \& Engineering \\ Australian National University \\ Email: jennifer.wu@rsise.anu.edu.au, thushara.abhayapala@anu.edu.au
}

\begin{abstract}
Reproduction of a soundfield is a fundamental problem in acoustic signal processing. A common approach is to use an array of loudspeakers to reproduce the desired field where the least square method is used to calculate the loudspeaker weights. However, the least square method involves matrix inversion which may lead to errors if the matrix is poorly conditioned. In this paper, we derive a new theoretical continuous loudspeaker method to obtain the loudspeaker aperture function in order to avoid matrix inversion. In addition, the aperture function obtained through continuous loudspeaker method reveals the underlying structure of the solution as a function of the desired soundfield, the loudspeaker positions and the frequency. Results are verified through simulations.
\end{abstract}

Index Terms - acoustic field, least square method, matrix inversion

\section{INTRODUCTION}

The ability to control the soundfield within a given region of space is a fundamental problem in acoustic signal processing. It is possible to reproduce a given soundfield using a set of loudspeakers. The first studies of soundfield reproduction were performed by Gerzon [1], in which he produced the firstorder spherical harmonics in terms of a plane wave soundfield at a single point in space. In [2] [3], Kirkeby proposed the least square techniques to determine the theoretical minimum number of loudspeakers required to produce a soundfield locally. Most recent works in soundfield reproduction such as [4], [5], [6], [7], [8] are based on the least square method due to the advantage that the sound is matched over a region of space rather than at a single point. However, the least square approach involves a matrix inversion, if the matrix is poorly conditioned, the solution may not exist. The condition number of the matrix dictates the sensitivity of errors of the resulting solution [9]. To avoid this, we apply the concept of continuous loudspeaker which has an aperture that is a function of space variables and frequency, and we derive an approach

Yan Jennifer WU's research work is funded through National ICT Australia. to obtain the loudspeaker aperture function without using matrix inversion. Then we approximate the continuous aperture by a finite number of discrete loudspeakers to produce the desired soundfield. In addition, the aperture function obtained through the continuous loudspeaker method reveals the underlying structure of the solution as a function of the desired soundfield, the loudspeaker positions and the frequency.

In [4], some fundamental performance limits for planewave soundfield reproduction in free space were developed using spherical harmonics analysis. However, it also used least square method to find the loudspeaker weights. In this paper, we seek to use the concept of continuous loudspeaker method to derive the performance bounds on the 2D planewave soundfield reproduction in free space (i.e. we ignore the effect of reverberation). Specifically, the relationships between the number of loudspeakers, the size of the reproduction region, the frequency range, and the desired accuracy are in correspondence with the results obtained in [4]. Simulation results demonstrate the favorable performance.

\section{SYSTEM MODEL}

In this paper, we concentrate on 2D or height invariant soundfield. Let the radius of the circular spatial zone be $r$, whose origin is at $O$ as shown in Figure 1. The loudspeakers are placed on the circle with angle $\phi$ and radius $R>r$. The weight of a particular loudspeaker located at angle $\phi$ is denoted as $\rho(\phi, k)$, where $k=2 \pi f / c$ is the wavenumber, $f$ is the frequency and $c$ is the speed of sound propagation of 340 $\mathrm{m} / \mathrm{s}$. Throughout this paper, we use $k$ instead of $f$ to represent frequency since we assume constant $c . k_{l}$ and $k_{u}$ are used to represent the lower limit and upper limit of the desired frequency band respectively. Vectors are represented by lower case bold face, e.g. $\boldsymbol{x}$. A unit vector in the direction $\boldsymbol{x}$ is denoted by $\hat{\boldsymbol{x}}$, i.e., $\hat{\boldsymbol{x}}=\boldsymbol{x} /|\boldsymbol{x}|$.

\subsection{Truncation Theorem}

We can represent an arbitrary 2D (height invariant) soundfield $S(\boldsymbol{x}, k)$ generated by any number of sound sources outside of 


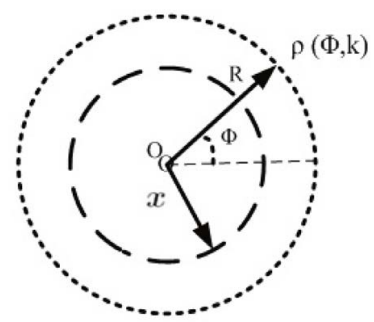

Fig. 1. System model

a region of radius $R$, having the following representation:

$$
S(\boldsymbol{x}, k)=\sum_{m=-\infty}^{\infty} \alpha_{m}(k) J_{m}(k\|\boldsymbol{x}\|) e^{i m \phi_{x}}
$$

where $J_{m}(\cdot)$ are the Bessel functions of order $m$, and $\alpha_{m}(k)$ are a set of coefficients for the sound field. Note, the representation (1) is in a form of a Fourier series expansion. The general representation of an arbitrary soundfield (1) can be constructed by solving the Helmholtz wave equation in cylindrical coordinate system [10, p.67]. Note that any arbitrary soundfield is a weighted sum of basis functions $J_{m}(k\|\boldsymbol{x}\|) e^{i m \phi_{x}}$, where $m$ is referred to as the mode.

The representation (1) has an infinite number of orthogonal modes, however, we can truncate this series expansion to a finite number due to the properties of the Bessel functions and the fact that the soundfield has to be upper bounded by a spatial region where all sources are outside [11]. Hence, (1) can be truncated to $|m| \leq M$ terms as:

$$
S_{M}(\boldsymbol{x}, k)=\sum_{m=-M}^{M} \alpha_{m}(k) J_{m}(k\|\boldsymbol{x}\|) e^{i m \phi_{x}},
$$

where the normalized truncation error is upper bounded as

$$
\epsilon_{M}(\boldsymbol{x})=\frac{\left|S(\boldsymbol{x}, k)-S_{M}(\boldsymbol{x}, k)\right|}{2 \pi|S(\mathbf{0}, k)|} \leq \eta e^{-\delta},
$$

provided that the truncation length is chosen as $M=\lceil e\|\boldsymbol{x}\| k / 2\rceil$ where $\eta \approx 0.16127$ and $\delta$ is a positive integer [11].

\subsection{Desired Soundfield}

We apply the truncation theorem for the desired soundfield. The desired soundfield at a point $\boldsymbol{x} \equiv(\|\boldsymbol{x}\|, \phi)$ from $O$ now becomes

$$
S^{\mathrm{d}}(\boldsymbol{x}, k)=\sum_{m=-M}^{M} \alpha_{m}^{(\mathrm{d})}(k) J_{m}(k\|\boldsymbol{x}\|) e^{i m \phi_{x}},
$$

where $\alpha_{m}^{(\mathrm{d})}(k)$ uniquely represents the desired field. Thus, the problem we consider in this paper is follows: Given a soundfield by its components $\alpha_{m}^{(d)}(k)$ for $m=-M, \ldots, M$, and $k$ $\in\left[k_{l}, k_{u}\right]$, how can we reconstruct the field in a spatial region of radius $r$ ?

\subsection{Actual Soundfield}

Assume that no loudspeaker is placed inside the spatial zone of interest. Let $\rho(\phi, k)$ be the aperture function of the circular continuous loudspeaker with radius $R$ to achieve the desired field. Thus, the reproduced soundfield within the circular zone at a point $\boldsymbol{x}$ from $O$ is given by

$$
S^{\mathrm{a}}(\boldsymbol{x}, k)=\int \rho(\phi, k) \frac{i}{4} H_{0}^{(1)}(k\|R \hat{\boldsymbol{\phi}}-\boldsymbol{x}\|) d \phi_{x} .
$$

Here we have assumed that the loudspeakers are infinitely long point cylinders (far-field sources). Note that the fundamental solution to the Helmholtz wave equation [10] in 2D is $(i / 4) H_{0}^{(1)}(k\|R \hat{\boldsymbol{\phi}}-\boldsymbol{x}\|)$, where the source is at $R \hat{\boldsymbol{\phi}}$ and the observation point is at $\boldsymbol{x}$, and $H_{m}^{(1)}(\cdot)$ is the Hankel function of the first kind ${ }^{1}$.

Provided $R>\|\boldsymbol{x}\|$, we can use the addition theorem for cylindrical harmonics [10] to write

$$
H_{0}^{(1)}(k\|R \hat{\boldsymbol{\phi}}-\boldsymbol{x}\|)=\sum_{m=-\infty}^{\infty} H_{m}^{(1)}(k\|R\|) e^{-i m \boldsymbol{\phi}} J_{m}(k|\boldsymbol{x}|) e^{i m \phi_{x}}
$$

We also use Fourier series expansion to express

$$
\rho(\phi, k)=\sum_{m=-\infty}^{\infty} \beta_{m}(k) e^{i m \phi},
$$

where $\beta_{m}(k)$ are Fourier coefficients.

By substituting (6) and (7) into (5),we get

$$
S^{\mathrm{a}}(\boldsymbol{x}, k)=\sum_{m=-\infty}^{\infty} \beta_{m}(k) \frac{i}{2} \pi H_{m}^{(1)}(k\|R\|) J_{m}(k\|\boldsymbol{x}\|) e^{i m \phi_{x}} .
$$

Note that $S^{\mathrm{a}}(\boldsymbol{x}, k)$ is a weighted sum of basis functions $J_{m}(k\|\boldsymbol{x}\|) e^{i m \phi_{x}}$, where the m-mode weight is $\beta_{m}(k) \frac{i}{2} \pi H_{m}^{(1)}(k\|R\|)$.

\section{CONTINUOUS LOUDSPEAKER DESIGN}

To design the loudspeaker aperture function, we equate the desired soundfield (4) to the actual soundfield (8). i.e,

$$
S^{\mathrm{d}}(\boldsymbol{x}, k)=S^{\mathrm{a}}(\boldsymbol{x}, k),
$$

for $\boldsymbol{x} \in$ desired region, $k \in\left[k_{l}, k_{u}\right]$.

Using (4) and (8), we have for $m=-M, \ldots, M$,

$$
\beta_{m}(k)=\frac{2}{i \pi H_{m}^{(1)}(k\|R\|)} \alpha_{m}^{(\mathrm{d})}(k) .
$$

We state this result as a theorem for reconstructing a given soundfield using a theoretical continuous circular loudspeaker:

\footnotetext{
${ }^{1}$ This is different from spherical wave fronts from a point source in $3 \mathrm{D}$, where the fundamental solution to the wave equation in $3 \mathrm{D}$ is $\exp (i\|R \hat{\boldsymbol{\phi}}-\boldsymbol{x}\|) /\|R \hat{\boldsymbol{\phi}}-\boldsymbol{x}\|$.
} 
Theorem 1 If a desired soundfield in a $2 D$ spatial region is given by the cylindrical harmonics of the soundfield $\alpha_{m}^{(d)}(k)$, for $m=-M, \ldots, M$, and frequency band $k \in\left[k_{l}, k_{u}\right]$, then the aperture function of the theoretical continuous circular loudspeaker of radius $R$ to reconstruct the soundfield is:

$$
\rho(\phi, k)=\sum_{m=-M}^{M} \frac{2}{i \pi H_{m}^{(1)}(k\|R\|)} \alpha_{m}^{(d)}(k) e^{i m \phi} .
$$

\section{DISCRETE LOUDSPEAKER ARRAY DESIGN}

We will now address the engineering problem of physically reproducing the soundfield using an array of loudspeakers. We began with the concept of continuous loudspeaker, in order to develop an exact relationship between the actual soundfield and the aperture function. The aperture function of the continuous loudspeaker will now be approximated by a discrete loudspeaker array to permit practical implementation.

By applying sampling theorem to (11), we can observe that if we have $Q>2 M$ points (loudspeakers) on the circle, then we can accurately reproduce $\rho(\phi, k)$ from its samples $\rho\left(\phi_{q}, k\right), q=1, \ldots, Q$. We assume these loudspeakers are equally $\triangle \phi$ spaced on a circle of radius $R$, then the aperture function at the $q$ th loudspeaker now becomes

$$
w_{q}(k) \approx \rho\left(\phi_{q}, k\right) \triangle \phi .
$$

Hence, the aperture function of the continuous loudspeaker is approximated to a discrete loudspeaker array by the following theorem:

Theorem 2 If a desired soundfield in a $2 D$ spatial region is given by the cylindrical harmonics of the sound field $\alpha_{m}^{(d)}(k)$, for $m=-M, \ldots, M$, and frequency band $k \in\left[k_{l}, k_{u}\right]$, we can use $Q$ discrete loudspeakers equally spaced on a circle of radius $R$, provided $Q>2 M$. Thus the weight at Qth loudspeaker to reconstruct the soundfield is:

$$
w_{q}(k)=\sum_{m=-M}^{M} \frac{2}{i \pi H_{m}^{(1)}(k\|R\|)} \alpha_{m}^{(d)}(k) e^{i m \phi_{q}} \triangle \phi .
$$

Remarks for Theorem 2:

1. From (11) and (13), we notice that the underlying structure of the loudspeaker aperture function/weights is a function of desired field, loudspeaker positions and frequency.

2. We have explicit exact form expression for loudspeaker aperture function which are related to loudspeaker location $\left(R, \phi_{q}\right)$ and model component of the desired soundfield $\alpha_{m}^{(\mathrm{d})}(k)$.

3. If the desired field is time varying, then there is no need to perform the matrix inversion at each time interval to update the loudspeaker weights. This leads to significant increased computational efficiency in a practical implementation.

\section{SIMULATION}

In this paper, we use a simple example to illustrate the ability to reproduce a $2 D$ sound field using the continuous loudspeaker method. We consider a circular reproduction region of radius $0.5 \mathrm{~m}$. The desired soundfield is monochromatic plane wave of frequency of $400 \mathrm{~Hz}$ arriving from $45^{\circ}$. This is equivalent to $k x=3.696$.

The rule of thumb proposed in [4] suggests $Q \geq 2 M+1$, where $M=\lceil k x\rceil$. This agrees with the sampling theorem to discretize the continuous aperture $Q>2 M$. In this case, we choose $M=4$, thus requiring $Q=9$ loudspeakers. The loudspeakers are equally spaced on a circle of $r=1 \mathrm{~m}$. The loudspeaker weights are calculated from (13), and the resulting reproduced field using continuous loudspeaker method is shown in Figure 2. It is calculated at $101 \times 101$ points and displayed as a "density plot" which means that the numerical values are represented by different shades of gray. The top two plots show the real and imaginary parts of the desired plane-wave field, and the bottom two plots show the field produced by the loudspeaker array. Figure 3 represents the 2D soundfield reproduction using least square method for comparison. The reproduced field in Figure 2 corresponds well to the desired field where the boundary of the reproduction area is indicated in the circle. It has the same performance as the sound field reproduced by the least square method. The reproduced error in this case is 0.0149 which agrees very well with the expected error of 0.04 referring to [4].
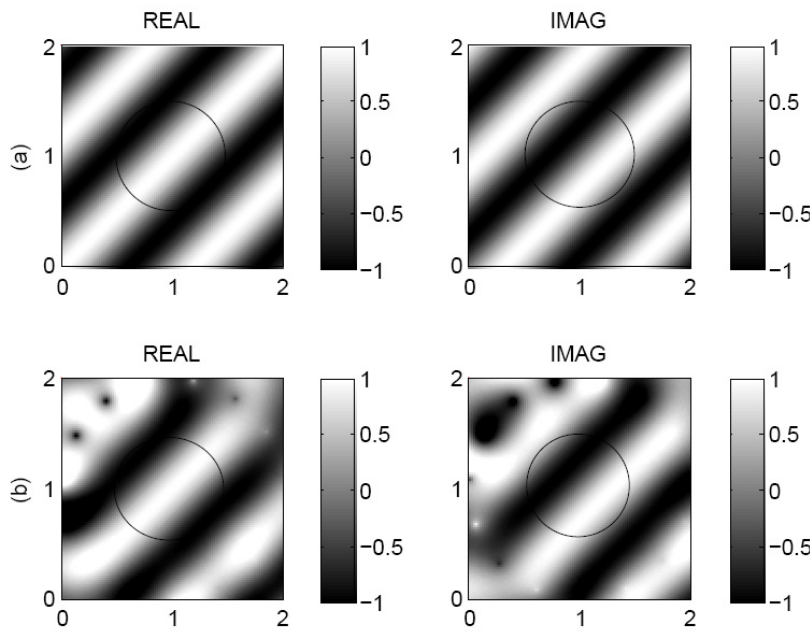

Fig. 2. Reproduction of a 2D plane-wave using continuous loudspeaker method (a) desired field (b) reproduction field

\subsection{Reproduction error}

We define the reproduction error as:

$$
\epsilon_{M}(k x)=\frac{\int\left\|S^{\mathrm{d}}(\boldsymbol{x}, k)-S^{\mathrm{a}}(\boldsymbol{x}, k)\right\|^{2} d \phi_{x}}{\int\left\|S^{\mathrm{d}}(\boldsymbol{x}, k)\right\|^{2} d \phi_{x}}
$$



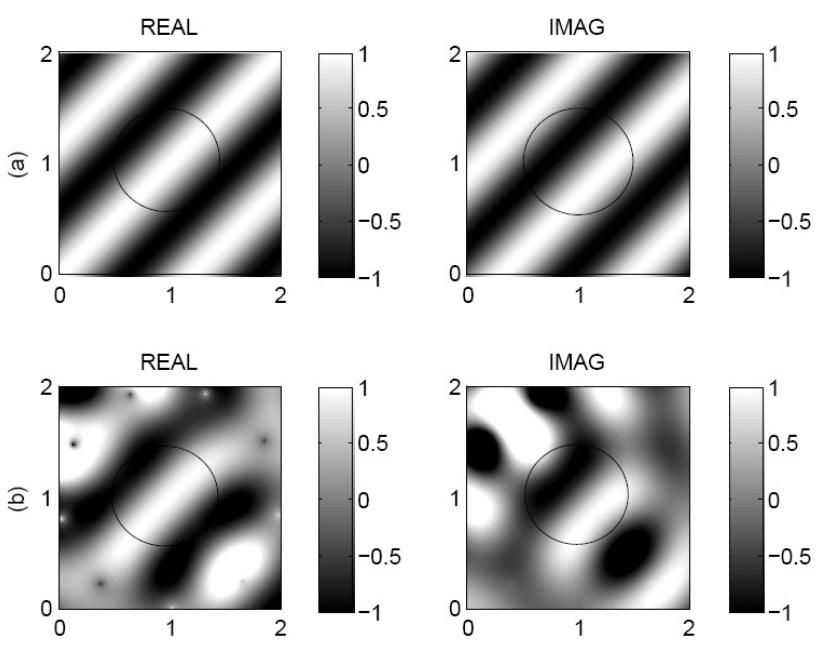

Fig. 3. Reproduction of a 2D plane-wave using least square method (a) desired field (b) reproduction field

The reproduced error for different $k r$ are shown in Figure 4. The reproduced error decreases with the increase of the number of loudspeakers. We notice when $Q>2\lceil k r\rceil$, the reproduced error is less than 0.04. Once $Q$ is beyond this threshold, a larger number of loudspeakers only marginally increases the reproduction accuracy. This validates the rule of thumb proposed in [11] for choosing the required expansion order.

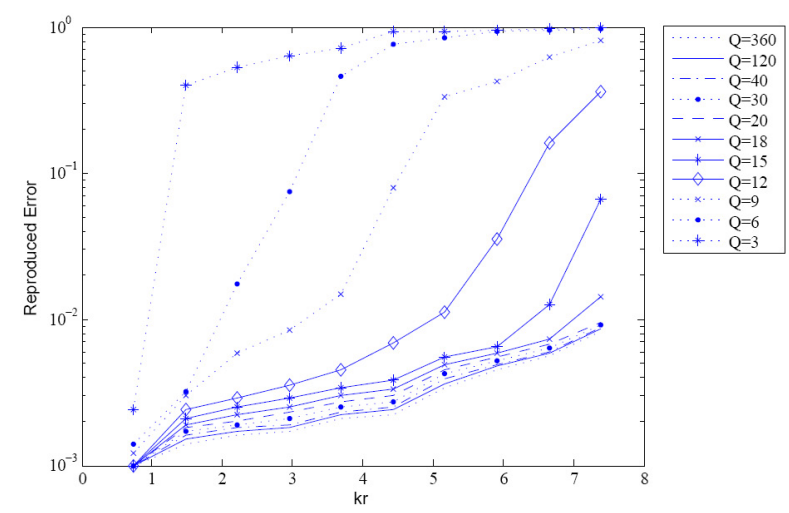

Fig. 4. Reproduction error for a 2-D plane-wave field as function of $k r$ for different number of loudspeakers $Q$

\section{CONCLUSION}

In this paper, we propose the theoretical continuous loudspeaker approach to calculate the loudspeaker aperture function in order to avoid matrix inversion required in the leastsquare methods. The underlying structure of the loudspeaker aperture function is a function of the desired field and the loudspeaker positions. We have shown that for a wavenumber $k$, we can accurately reproduce the sound field within radius $r$ by using a discrete number of $Q>2\lceil k r\rceil$ loudspeakers. This continuous loudspeaker approach can be applied to the soundfield reproduction in reverberant environment and also can be extended for the $3 D$ soundfield reproduction in the future work.

\section{REFERENCES}

[1] M.A. Gerzon, "Periphony: With-height south field reproduction," J. Audio. Eng. Soc, vol. 21, pp. 2-10, January 1973.

[2] O. Kirkeby and P.A. Nelson, "Reproduction of plane wave sound fields," J. Acoust. Soc. Am, 1993.

[3] O. Kirkeby, P.A. Nelson, F. Orduna-Bustamante, and H. Hamada, "Local sound field reproduction using digital signal processing," J. Acoust. Soc. Am, vol. 100, no. 3, pp. 1584-1593, September 1996.

[4] D.B. Ward and T.D. Abhayapala, "Reproduction of plane wave sound field using an array of loudspeakers," IEEE Trans. Speech, Audio Process., vol. 9, pp. 697707, September 2001.

[5] M. Poletti, "Robust two-dimensional surround sound reproduction for nonuniform loudspeaker layouts," J. Audio. Eng. Soc, vol. 55, no. 7/8, pp. 598-610, July/August 2007.

[6] T. Betlehem and T.D. Abhayapala, "Theory and design of sound field reproduction in reverberant rooms," $J$. Acoust. Soc. Am, vol. 117, no. 4, pp. 2100-2111, April 2005.

[7] R. Bruno, A. Laborie, and S. Montoya, "Reproducing multichannel sound on any loudspeaker layout," in Proc. 118th AES Convention, Barcelona, Spain, May.

[8] J. Merimaa and V. Pulkki, "Spatial impulse response rendering i: Analysis and synthesis," J. Audio. Eng. Soc., vol. 53, no. 12, pp. 1115-1127, 2005.

[9] P.A. Nelson and J.W. Rose, "Errors in two-point sound reproduction," J. Acoust. Soc. Am, vol. 118, no. 1, pp. 193-204, July 2005.

[10] D. Colton and R. Kress, Inverse Acoustic and Electromagnetic Scattering Theory, Springer, New York, second edition edition, 1998.

[11] R.A. Kennedy, P. Sadeghi, T.D. Abhayapala, and H.M. Jones, "Intrinsic limits of dimensionality and richness in random multipath fields," IEEE Trans. Sig. Process., 2007. 